\title{
When the Trauma is Serious
}

\author{
Dra Mirta D Ambra $\mathbf{M}^{1 *}$, Ilie Vasiliev ${ }^{2}$ and Valeri Shevchenko ${ }^{3}$ \\ ${ }^{1}$ University of Buenos Aires, Argentina \\ ${ }^{2,3}$ World American Medical Sciences, Argentina
}

*Corresponding author: Dra Mirta D Ambra M, Board Executive WAMS, Professor Public Health Specialist Ministry of Health, University of Buenos Aires, Argentina.

To Cite This Article: : Dra Mirta D Ambra M. When the Trauma is Serious. Am J Biomed Sci \& Res. 2019 - 2(6). AJBSR.MS.ID.000616.

DOI: 10.34297/AJBSR.2019.02.000616

Received: April 01, 2019 | Published: May 06, 2019

\begin{abstract}
Summary
a. The decisive and advanced position on emergency surgery continues to be the guiding principle in the initial treatment of serious injuries.

b. Some interventions are mechanical (tourniquet), others are pharmacological (antifibrinolytic treatment) and others require new ways of thinking (early damage control surgery).

c. From the first moment of the injury, the goal should be to achieve surgical arrest of the hemorrhage.

d. Damage reduction surgery is a technical strategy that prioritizes the arrest of bleeding, leaves the abdominal cavity with a temporary closure and postpones all other maneuvers and surgical reconstructions for further operations.

e. Most deaths due to massive hemorrhage occur within 30 minutes of the injury, therefore interventions should be immediate.

f. Normotensive patients should not receive resuscitation with fluids.

g. In hypotensive patients, wait until the systolic pressure approaches $80 \mathrm{~mm} \mathrm{Hg}$.

h. Blood products, especially when a massive transfusion is performed, should be used in all institutions that receive patients with

i. Aortic endovascular balloon resuscitation (REBOA) is a rapidly emerging technique to stop non-compressible infradiaphragmatic intracavitary hemorrhage.
\end{abstract}

\section{Introduction}

Hippocrates wrote, "He who wishes to be a surgeon should go to war." It is regrettable that rapid progress in trauma care is often strongly linked to armed conflict. William Mayo, many centuries later, affirmed, "Medicine is the only victor in war"[1].

Injuries caused by war often led to innovations in trauma care and surgical creativity. Others evolved through the natural path of external review by experts, publication and acceptance by colleagues. Investigating the treatment of serious injuries is very difficult and innovations are often driven by necessity rather than the scientific method [2].

However, the current rates of survival after severe trauma are the highest in the history of medicine and the recent improvements in medical care are attributable in part to the almost two decades of war on terrorism.

In the United States, injuries are still the main cause of death among people aged 1-44, highlighting that they are not just a problem of wars.
This article reviews the main advances in the care of patients with serious injuries. Practicing the best evidence-based medicine for trauma care often requires imperfect decisions based on incomplete and evolving information. The decisive and advanced position about emergency surgery continues to be the guiding principle [3].

\section{Turnstiles}

At the beginning of the war against terrorism, the rate of death due to bleeding of limbs and joint injuries was extremely high, despite a 1996 report on military medicine where the authors recognized the need to use turnstiles on the ground to the lifethreatening hemorrhages of the extremities. The improvised turnstiles were frequent, although very ineffective.

The military recognized the need for better control of limb bleeding at the site of the injury and commercial devices to stop the bleeding of limbs became common on the battlefield, along with universal training on how to use them. These changes greatly reduced deaths from limb exsanguination [4]. 
In previous wars the turnstiles fell into disrepute due to delayed evacuation and consequent loss of limbs due to ischemia. However, in current conflicts evacuation times are much shorter and this complication is rare. Topical hemostatic bandages also appeared to prevent bleeding of the limbs and joints.

Upon the return of the soldiers from the front, commercial turnstiles, together with adequate training for their use, became popular for common wounds, stimulated in part by incidents with multiple deaths. These terrible episodes warned about the need to quickly stop the hemorrhaging of the extremities.

The available data indicate that all emergency services should adopt this intensive strategy to mitigate bleeding at the site of the injury. Once applied, the tourniquet must remain tight until it is safe to remove it to evaluate the patient in a hospital with surgical capacity [5].

\section{Antifibrinolytic Treatment}

Although the coagulopathy of the traumatisms is not completely known, it is known that one of its components is malignant hyperfibrinolysis. Fibrinolysis is a normal intravascular process that maintains the proper balance with thrombosis.

After a severe injury, a hyper fibrinolytic state develops in some patients, where the thrombus is endogenously lysed more rapidly than it can be formed. This alteration can aggravate the hemorrhage and contribute to death [6].

Tranexamic acid, an antifibrinolytic drug, was used for decades to mitigate postpartum hemorrhage. However, its use to treat or prevent hyperfibrinolysis in trauma patients was recognized only a few years ago.

Treatment with tranexamic acid is simple and its effect is considerable if it is given as indicated in patients with more severe injuries. (1 $\mathrm{g}$ administered as an intravenous bolus for 10 minutes, followed by infusion of $1 \mathrm{~g}$ intravenously for 8 hours, administering the first dose within 3 hours after the trauma).

For these reasons tranexamic acid was adopted as a treatment on the battlefield and is gaining acceptance in the US. The sooner after the trauma treatment is administered, the greater its utility with respect to mortality rates. One must be careful, however, since the administration of tranexamic acid more than 3 hours after the trauma can increase the risk of death.

The use of this drug should also be incorporated into mass transfusion programs. There were no problems attributed to its universal adoption as an initial intervention for patients with suspected severe bleeding.

Although it is not an antifibrinolytic intervention directly, the administration of plasma during transfer to the hospital improves coagulation and decreases overall mortality [7].

\section{Permissive Hypotension}

Normotensive patients should not receive resuscitation with fluids. A century ago, Walter Cannon stated that sources of hemorrhage that are inaccessible or that cannot be stopped should not be treated with intravenous fluids until the time of surgery. It took another 76 years to validate this scientifically in a careful study. It is regrettable that the strategy of waiting for resuscitation with liquids until stopping the bleeding has spread slowly.

For most of the twentieth century, allowing patients with trauma to continue hypotensive until the surgical intervention violated an important principle of resuscitation with crystalloid solutions. The frequent practice of administering 2 liters of crystalloids in patients with trauma and hypotension worsens coagulopathy and acidosis and should be abandoned.

Normotensive patients should not receive resuscitation with liquids, while for this resuscitation in hypotensive patients should wait until the systolic pressure approaches $80 \mathrm{~mm} \mathrm{Hg}$. At that time, small volume boluses of blood or plasma should be carefully administered (250 to $500 \mathrm{ml}$ ) in order to maintain the systolic pressure between 80 - and $90-\mathrm{mm} \mathrm{Hg}$.

The wars in the Middle East favored the use and acceptance of permissive hypotension. This approach, in addition to insurance, can be very beneficial for patients with penetrating or blunt injuries.

The safe limits of permissive hypotension are not known, but administration of a large volume of intravenous fluids prior to surgical arrest of the hemorrhage is dangerous and should not be performed.

\section{Damage Reduction Surgery}

Damage reduction surgery is a technical strategy to stop massive hemorrhage. This approach prioritizes the arrest of bleeding and the reduction of contamination in the initial surgical intervention and involves leaving the abdominal cavity with a temporary closure and postponing all other maneuvers and surgical reconstructions for further operations.

Sometimes called "surgery in several times", this intervention favors survival in patients with the most severe injuries and the greatest hemorrhages. Some patients must undergo successive operations for several days in order to avoid the trauma of a prolonged operation that involves extensive hemorrhage.

Between each operation the patient will be in the intensive care unit (ICU), where he / she is treated with attention to the resuscitation, the resolution of the acidosis, the maintenance of the normothermia and the elimination of the coagulopathy, usually with the use of sedation and assisted breathing.

In the subsequent operations the definitive surgical reconstruction is carried out according to the physiological tolerance of the patient and the abdomen is closed as soon as the reconstruction is completed. This strategy has been adopted by trauma centers in many countries, including those with adequate medical resources.

At present, it is considered the standard of care for patients with the most serious injuries undergoing surgery due to massive hemorrhage. Its use directly attenuates the vicious circle of hypothermia, acidosis and coagulopathy [8]. 
The next operation will be carried out within 24 hours of the end of the first and each subsequent operation must be performed within 24 hours of the previous one in order to improve the chances of primary fascial closure. Surgical attempts at primary fascial closure should be made daily, since the physical examination cannot predict when the fascia will close.

The use of a vacuum device for temporary abdominal closure and the early use of temporary neuromuscular block improves the rates of primary fascial closure and should be considered if they are not contraindicated. Patients for damage reduction surgery should be carefully selected, since repeated operations increase morbidity among patients with only moderate injuries.

\section{The Golden Hour}

During the First World War, the French published the first scientific report on the importance of time in the treatment of post-trauma shock. The recognition that the intervention should be carried out quickly led to the development of emergency medical systems.

The nickname "The golden hour" summed up this approach for public policy makers, although it overlooks the reality that most deaths due to thoracic hemorrhage occur within 30 minutes of the injury. More recently, data from the wars in Iraq and Afghanistan suggest that survival on the battlefield after trauma is closely linked to the interval between injury and evacuation for the first surgical intervention. This motivated the order of the Ministry of Defense of the E.E.U.U. to evacuate by helicopter all the wounded in combat within 60 minutes of the injury. This order served to save lives [8].

Knowing that trauma is sensitive to time remains of the utmost importance, since the interval between trauma and surgical intervention determines the results, both on the battlefield and outside it. Surgical intervention, however, should not be confused with triage and resuscitation.

The latter is not a substitute for stopping bleeding and caution should be exercised when resuscitative measures are initiated without a plan for surgical detention of the hemorrhage The main purpose of the golden hour concept is to direct all efforts toward early arrest of bleeding, including initial assistance, triage, rapid evacuation and resuscitation.

The main purpose of the golden hour concept is to direct all efforts toward early arrest of bleeding, including initial assistance, triage, rapid evacuation and resuscitation.

From the first moment of the injury, the objective should be to achieve the surgical arrest of the hemorrhage. All other maneuvers are in support of this main objective.

\section{Possible Interventions During the Golden Hour}

The main objective of the concept of the golden hour is to achieve early control of the hemorrhage. The prehospital maneuvers and in the hospital for this purpose are initial assistance, triage, rapid evacuation and resuscitation [9].

FAST is the ultrasound of the abdominal cavity and the pericardium during the initial phase of medical care.
REBOA is the resuscitation with endovascular aortic balloon

\section{Massive Transfusion}

Patients with hemorrhage need blood. The use of other intravenous fluids as a means of resuscitation worsens the results. Whole blood or a substitute like this should be used for resuscitation, with simultaneous initiation of maneuvers to stop the bleeding.

Blood products, especially in the context of an organized mass transfusion protocol, with emphasis on a high proportion of erythrocyte concentrate plasma should be used in all institutions receiving patients with trauma [10].

Blood products are refrigerated for storage and should be warmed to body temperature using a liquid heating device during resuscitation. This is an important point because the transfusion of blood products in a patient with trauma and hemorrhage contributes to iatrogenic hypothermia and coagulopathy.

The speed of administration should be proportional to the degree of shock and follow the principles of permissive hypotension. The blood products should be administered at the highest possible speed in order to respect the principles of hypotensive resuscitation, with the aim of a systolic pressure of $80 \mathrm{~mm} \mathrm{Hg}$ during damage reduction surgery [11].

\section{Commentary}

The initial care of the patient with serious injuries has changed markedly in recent decades, largely stimulated by the experience of recent wars.

1. Continuous advances in trauma care outside armed conflicts require national commitment to research, especially in some areas with great promise, but little data or limited acceptance.

2. Injury care requires an extremely intensive surgical approach, despite incomplete, imperfect and rapidly changing information.

3. This approach should be used immediately, starting at the site of the injury.

4. It is necessary to adopt these advances more widely in order to improve the survival of patients with more serious injuries, especially those with massive hemorrhage.

\section{References}

1. (1817) The Aphorisms of Hippocrates. Collins, New York, USA.

2. Rasmussen TE, Kellermann AL (2016) Wartime lessons-shaping a national trauma action plan. N Engl J Med 375(17): 1612-1615.

3. Eastridge BJ, Mabry RL, Seguin P, Moores L, Blackbourne LH, et al. (2012) Death on the battlefield (2001- 2011): implications for the future of combat casualty care. J Trauma Acute Care Surg 73(5): S431-S437.

4. Butler FK, Hagmann J, Butler EG (1996) Tactical combat casualty care in special operations. Mil Med 161: 3-16.

5. Kragh JF, Walters TJ, Baer DG, Salinas J, Holcomb JB, et al. (2009) Survival with emergency tourniquet use to stop bleeding in major limb trauma. Ann Surg 249(1): 1-7. 
6. Bennett BL, Littlejohn LF, Kheirabadi BS (2014) Management of external hemorrhage in Tactical Combat Casualty Care: Chitosan-based hemostatic gauze dressings-TCCC guidelines-change 13-05. J Spec Oper Med 14(3): 40-57.

7. King DR, Larentzakis A, Ramly EP (2015) Tourniquet use at the Boston Marathon bombing lost in translation. J Trauma Acute Care Surg 78(3): 594-599.

8. Jacobs LM, McSwain N, Rotondo M, Wade D, Fabbri W, et al. (2013) Improving survival from active shooter events: the Hartford Consensus. Bull Am Coll Surg 74(6): 1399-1400.
9. Bulger EM, Snyder D, Schoelles K, Gotschall C, Dawson D, et al. (2014) An evidence-based prehospital guideline for external hemorrhage control: American College of Surgeons Committee on Trauma. Prehosp Emerg Care 18(2): 163-173.

10. Ives C, Inaba $\mathrm{K}$, Branco $\mathrm{BC}$, Okoye O, Schochl $\mathrm{H}$, et al. (2012) Hyperfibrinolysis elicited via thromboelastographic predicts mortality in trauma. J Am Coll Surg 215(4): 496-502.

11. Woman Trial Collaborators (2017) Effect of early tranexamic acid administration on mortality, hysterectomy, and other morbidities in women with post-partum hemorrhage (Woman): an international, randomized, blind, placebo-controlled trial. 389(10084): 2105-2116. 